\title{
Editorial Message: Special Issue on Advances Fuzzy methods on Control, Clustering and Decision-Making
}

\author{
Jin-Tsong Jeng ${ }^{1}$ - Chen-Chia Chuang ${ }^{2}$
}

Published online: 2 December 2021

(C) Taiwan Fuzzy Systems Association 2021

System Science and Engineering has emerged as a research field that covers a wide spectrum of modern technology. In recent, Advances Fuzzy methods on Control, Clustering and Decision Making have been the popular directions in System Science and Engineering. Advances Fuzzy methods on Control, Clustering and Decision Making on this special issue have Fuzzy Control Under Time-Varying Universe and Phase Optimization in Traffic Lights, The Fuzzy Control Approach for a Quadruped Robot Guide Dog, Wavelet K-Means Clustering and Fuzzy-Based Method for Segmenting MRI Images Depicting Parkinson's Disease, A General Transfer Learning-Based Gaussian Mixture Model for Clustering, A PROMETHEE II Approach Based on Probabilistic Hesitant Fuzzy Linguistic Information with Applications to Multi-Criteria Group Decision Making and An Extended Taxonomy Method Based on Normal T-Spherical Fuzzy Numbers for Multiple-Attribute Decision-Making. Besides, Advances Fuzzy methods on Control, Clustering and Decision Making have been successfully used in many areas, such as system control, industrial application, human resource management, forecasting of time-series, pattern recognition, medical image processing, system science and engineering and etc. The objective of this special issue is to explore novel Advances Fuzzy methods on Control, Clustering and Decision Making at The International Conference on System Science and Engineering 2020 (ICSSE 2020) that is an

Jin-Tsong Jeng

tsong@nfu.edu.tw

1 Department of Computer Science and Information Engineering, National Formosa University, Yunlin, Taiwan

2 Department of Electrical Engineering, National Ilan University, Ilan, Taiwan international conference that will take place in Sunport Hall Takamatsu, Kagawa, Japan during August 31September 3, 2020 with virtual conference and physical conference in National Taiwan University of Science and Technology, Taiwan. Papers submitted to the International Journal of Fuzzy Systems (IJFS) special issue were initially reviewed by the guest editor and accepted as the oral presentation. In accordance with the strict paper review procedure of IJFS, we invited IJFS associate editors, Prof. Shun-Feng Su, Prof. Jin-Tsong Jeng, Prof. Chen-Chia Chuang, Prof. Ching-Chih Tsai, Prof. Deng-Feng Li, Prof. Huchang Liao, Prof. Kao-Yi Shen, Prof. Chia-Feng Juang, Prof. Chun-Fei Hsu, Prof. Wen-Jer Chang, Prof. Yueying Wang, Prof. Wei Sun, Prof. TP Hong, Prof. Weiwei Che, Prof. Wei Sun, Prof. Rumei Li, Prof. I-Hsum Li, and Prof. Mei-Yung Chen as the reviewers of all the oral presentations with virtual/physical conference on ICSSE 2020. These papers have oral presentation with virtual/physical conference on a question-and-answer session with three reviewers and are open to all the audiences. Totally six papers were selected according to the review criterion on completeness and technical contributions and asked for quality improvements according to the reviewers' comments. Throughout the elaborative revisions by the authors, these six papers have finally been accepted for publication in the IJFS special issue Advances Fuzzy methods on Control, Clustering and Decision Making. As for the presented contents of these six papers in the special issue, we make brief introductions to their contributions on Advances Fuzzy methods on Control, Clustering and Decision Making that have one paper in this volume, this articles provide interesting and timely innovative results covering: An Extended Taxonomy Method Based on Normal T-Spherical Fuzzy Numbers for Multiple-Attribute Decision-Making. Three papers in volume 23(6), these articles 
provide interesting and timely innovative results covering: The Fuzzy Control Approach for a Quadruped Robot Guide Dog, Wavelet K-Means Clustering and Fuzzy-Based Method for Segmenting MRI Images Depicting Parkinson's Disease, and A PROMETHEE II Approach Based on Probabilistic Hesitant Fuzzy Linguistic Information with Applications to Multi-Criteria Group Decision Making. Two papers in volume 23(3), these articles provide interesting and timely innovative results covering: Fuzzy Control Under Time-Varying Universe and Phase Optimization in Traffic Lights, and A General Transfer Learning-Based
Gaussian Mixture Model for Clustering. These six papers bring a rich collection of Advances Fuzzy methods on Control, Clustering and Decision Making to illustrate the main technical achievements on the IJFS special issue of ICSSE 2020. Finally, I would like to acknowledge all the contributors to this special issue on the IJFS special issue of ICSSE 2020.

Guest Editor, IJFS Special Issue on Advances Fuzzy methods on Control, Clustering and Decision Making of ICSSE 2020. 